\title{
High ambient temperature and mortality: a review of epidemiologic studies from 200 I to 2008 Rupa Basu
}

Address: California Office of Environmental Hazard Assessment, Air Pollution Epidemiology Section, Oakland, California, USA Email: Rupa Basu - rbasu@oehha.ca.gov

Published: 16 September 2009

Environmental Health 2009, 8:40 doi:10.1 186/1476-069X-8-40

This article is available from: http://www.ehjournal.net/content/8/I/40

Basu; licensee BioMed Central Ltd.

This is an Open Access article distributed under the terms of the Creative Commons Attribution License (http://creativecommons.org/licenses/by/2.0), which permits unrestricted use, distribution, and reproduction in any medium, provided the original work is properly cited.

\begin{abstract}
Background: This review examines recent evidence on mortality from elevated ambient temperature for studies published from January 200I to December 2008.

Methods: PubMed was used to search for the following keywords: temperature, apparent temperature, heat, heat index, and mortality. The search was limited to the English language and epidemiologic studies. Studies that reported mortality counts or excess deaths following heat waves were excluded so that the focus remained on general ambient temperature and mortality in a variety of locations. Studies focusing on cold temperature effects were also excluded.
\end{abstract}

Results: Thirty-six total studies were presented in three tables: I) elevated ambient temperature and mortality; 2) air pollutants as confounders and/or effect modifiers of the elevated ambient temperature and mortality association; and 3) vulnerable subgroups of the elevated ambient temperature-mortality association. The evidence suggests that particulate matter with less than 10 um in aerodynamic diameter and ozone may confound the association, while ozone was an effect modifier in the warmer months in some locations. Nonetheless, the independent effect of temperature and mortality was withheld. Elevated temperature was associated with increased risk for those dying from cardiovascular, respiratory, cerebrovascular, and some specific cardiovascular diseases, such as ischemic heart disease, congestive heart failure, and myocardial infarction. Vulnerable subgroups also included: Black racial/ethnic group, women, those with lower socioeconomic status, and several age groups, particularly the elderly over 65 years of age as well as infants and young children.

Conclusion: Many of these outcomes and vulnerable subgroups have only been identified in recent studies and varied by location and study population. Thus, region-specific policies, especially in urban areas, are vital to the mitigation of heat-related deaths.

\section{Background}

Although many studies of temperature have been conducted in other disciplines such as climatology, they have received greater attention in epidemiology in the past few years. In 2002, a comprehensive epidemiologic review by Basu and Samet [1] summarized the findings from studies examining mortality from elevated ambient temperature and heat waves. Most of the evidence at that time was based on studies following heat waves. Several studies have been conducted more recently using modern statistical approaches, consisting primarily of the time-series and case-crossover approaches. While other reviews have been 
conducted more recently on heat and mortality, the focus has been on methodological issues and approaches [2] and on climatology [3], leaving a number of important epidemiologic studies excluded. Many studies of ambient temperature and mortality did not account for air pollutants, and in the previous review [1], it was not clear from the few studies conducted whether air pollutants acted as confounders, effect modifiers, or both. It is critical to separate the independent effects of both ambient temperature and air pollutants, since they may often influence each other on a daily basis. Thus, the actual association between ambient temperature and mortality can be observed, only after accounting for pollutants in the models with ambient temperature. Furthermore, demographic characteristics, such as poverty and age, can modify the severity of heat effects through various physiological and behavioral pathways. Thus, certain subgroups may be particularly vulnerable to heat effects, and identifying them for specific locations would be beneficial for targeting public health interventions.

Here, the epidemiologic evidence from the past decade of high ambient temperature and mortality is summarized, with a closer examination of studies of the potential effect of air pollution on the temperature-mortality association, as well as vulnerable subgroups. A general discussion of mortality displacement on the association between temperature and mortality is also included. Mortality displacement (also known as harvesting) refers to the phenomena suggesting that observed deaths from some environmental exposure, such as ambient temperature, occur in the most frail individuals whose deaths have only been brought forward by a few days.

\section{Methodological issues \\ Inclusion/exclusion criteria}

All studies included in this review were published in peerreviewed journals between January 2001 and December 2008. PubMed was used to search for the following keywords: temperature, apparent temperature, heat, heat index, and mortality. In addition, Table 2 had the keyword "air pollutants," "ozone," or "particulate matter" added, and Table 3 had "vulnerable", "susceptible subgroups/groups" added. The search was limited to the English language and epidemiologic studies. The review focused primarily on quantitative studies of ambient temperature, consisting of studies using the time-series and/or case-crossover methods. The outcomes from these studies generally reported a regression coefficient, relative risk (time series), odds ratio (case-crossover), or percent change in mortality, along with corresponding standard errors or confidence intervals. Studies that reported mortality counts or excess deaths following heat waves were excluded so that the focus remained on general ambient temperature exposure, rather than on short time periods.
Review articles or studies focusing on cold temperature effects were also excluded. Thirty-six studies published in peer-reviewed journals were selected for this epidemiologic review, with 52 total studies in the references that included a general discussion of temperature and mortality.

\section{Exposure assessment}

Temperature data are often measured near airport monitoring stations, and analyzed at the city or county level. Thus, misclassification of exposure may occur, especially for larger geographic areas. Also, measuring ambient temperature outside of urban areas may artificially reduce the temperature measurement, since urban areas often have higher temperatures than suburban or surrounding areas because of heat absorbed by buildings and roadways (known as the urban heat-island effect). However, since the bias should be non-differential (i.e., not different by county or other unit of analysis), the bias in the estimate would be toward the null, where the results would be underestimated. Misclassification of exposure may be reduced in future studies by using smaller buffer zones, such as five or ten kilometers around each monitor, if sufficient data are available.

Exposure to ambient temperature is often defined as some combined metric of temperature and relative humidity or dew point temperature, such as heat index, humidex, or apparent temperature, depending on the study location and author's preference. In addition, other variables, such as day of the week, time trend, and barometric pressure, are often added to the model. In some studies, air pollutants have been assessed as confounders or effect modifiers in an attempt to tease apart the independent effect of temperature. A monitor or an average of monitors is often used to characterize exposure for a county, or a given distance from the home address using geospatial coding. Many investigators relied on mean daily average to classify exposure, although others used maximum or minimum temperature to capture daytime and nighttime exposures, respectively, since those have also been shown to play a role in heat-related mortality. Because the effect of temperature has been found to be immediate (i.e., same lagged day), exposure can be characterized by the place of death.

\section{Case selection}

Heat-related mortality is often underestimated, and since a systematic definition still does not exist, it may only be indicated when heat waves occur, resulting in a signal detection bias. Thus, investigators often use all-cause mortality excluding mortality due to accidents, or other related outcome, such as mortality from cardiovascular or respiratory diseases in epidemiologic studies of heat or elevated ambient temperature. The underlying cause of 
death is usually used for epidemiologic studies and should be sufficient for characterizing the temperaturemortality association, although associated causes of death can also be used.

The studies to date are often limited by information provided by the death certificate data. For example, information on income level, poverty, or air conditioning use is not offered on the individual level, so it is difficult to examine socioeconomic status. Thus, gathering data on individual characteristics, as has been done in a previous study [4], would be informative. In addition, medication use, time-activity patterns, and biologic mechanisms could be further understood.

\section{Study design}

Most of the studies conducted in the past decade rely on the time-series or case-crossover study designs, with the exception of the studies of the 2003 European heat wave, which are not included in this review. Regardless of the method chosen, the time-series and case-crossover study designs should yield similar results, as has been shown in some temperature-mortality studies [5-7].

The time-series is a widely accepted approach in both air pollution and temperature studies. The time-series study generally encompasses large populations in multiple geographic areas over a given time period. Mortality counts or rates are compared to exposure measurements collected at regular time intervals (e.g., daily, weekly). Seasonality and other confounding factors that fluctuate over time are accounted for by adding the covariates to the model (i.e., day of the week, air pollutants) and using smoothing functions to the model using specified degrees of freedom.

The case-crossover design has been employed more recently, gaining widespread popularity for studying air pollution and temperature in the past decade. This study design is similar to a matched case-control study; however, each case in the case-crossover study serves as his/her own control. Thus, biases due to measured and unmeasured confounders, such as genetics, health behaviors, and physiologic differences, are controlled for by study design. The case-crossover study design has been refined since its introduction in 1991, from the unidirectional, to the bidirectional, and most recently, to the time-stratified approach. The time-stratified approach limits the bias from selecting control periods only previously to the case period (unidirectional), or from not selecting control periods at random from the time at which the case occurred (bidirectional). Most commonly, control periods are selected within the same month and the same year that the case period occurred in the time-stratified approach to inherently minimize biases that may occur from time trends. Day of the week is also matched for by study design by choosing control periods every seven days, or may be added to the model as an indicator variable, especially if data are sparse (e.g., if using particulate matter in the model, data are often collected every third day, and thus, would warrant control periods to be selected every third day). In the studies listed in the following tables, all case-crossover studies used the timestratified approach.

Multi-city analyses are preferred, since bias from a single city analysis may result and the findings from multiple areas may be more generalizable [6]. Thus, city or countylevel estimates are usually combined into one overall estimate using meta-analytic techniques using a random effects model.

\section{Mortality displacement}

In epidemiologic studies of temperature or air pollution and mortality or morbidity, mortality displacement/harvesting have been addressed using several methods [8-13]. Among the more intuitive approaches, one can examine very long cumulative averages (i.e., 20 to 40 days of exposure) to determine whether a positive association found over the first few days is offset by a negative association over subsequent days. This would suggest that a pool of frail individuals was the only or major subgroup that was impacted by the exposure. However, if harvesting is not found, then the exposure under study is a real public health issue.

In studies of temperature and mortality, very few studies have addressed the harvesting issue. The evidence is mixed and may depend on: (1) whether one is examining heat waves versus a more general rise in temperature; (2) the study design and lag structure used for temperature effects; (3) the potential interactions with air pollution; (4) the baseline health status of the population; (5) the population at risk; and (6) other local factors that might determine vulnerability.

\section{Summary of Studies}

Fourteen studies were epidemiologic studies of ambient temperature and mortality, while 14 other studies considered air pollutants as potential confounders/effect modifiers, and six considered vulnerable subgroups. Most of these studies used either the time-series method $(n=29)$, while fewer used the case-crossover approach $(n=10)$. Eleven studies were conducted in the US. Ten studies were published using European data, three in Latin America, three in Australia, two in Canada, and elsewhere. The studies are all summarized in the following Tables 1 to 3 by year of publication, with the most recent studies first, followed by alphabetical order of the first author's last name. Since some studies included an examination of 
general ambient temperature and mortality, accounted for air pollutants, and/or identified vulnerable subgroups, the same study may be listed in multiple tables with the relevant results.

\section{General Ambient Temperature and Mortality}

In Table 1, the recent studies of high ambient temperature and mortality are summarized. To focus on the effects of warmer temperatures, most investigators limited their data above a threshold value, or have compared effect estimates from temperatures above a threshold value to another lower value. The threshold value is often based on some percentile of the data (i.e., $90^{\text {th }}$ or $95^{\text {th }}$ percentile), after visual inspection of the exposure-response curves or by mathematical (i.e., through derivatives) or statistical (i.e., by maximum likelihood) methods. The data are often limited to the summer months or warm season to establish heat effects. Limiting the data to summer months or the warm season is also employed to exclude possible (negative or positive) effects from cold temperatures on mortality in the attempt to estimate the actual risk from heat effects. Because of these several classifications of temperature exposure, it is difficult to directly compare the values resulting from these studies. However, few comparisons can be made. For example, in Europe and Korea, where different levels of temperature and humidity were experienced, the mortality estimates above a threshold of $\left(23.3-29.7^{\circ} \mathrm{C}\right)$ resulted in different effect estimates [12]. With similar threshold values in the Mediterranean $\left(29.4^{\circ} \mathrm{C}\right)$ and Korea $\left(27-29.7^{\circ} \mathrm{C}\right)$, a $1^{\circ} \mathrm{C}$ increase of apparent temperature corresponded to a $3.12 \%$ increase in daily mortality in Mediterranean cities, and a much higher effect in Korea (6.73\%-16.3\% in six cities) for a similar time period. Two recent studies conducted by Basu et al. $[6,14]$ and Zanobetti and Schwartz [7] using identical methods suggested that the effect estimates throughout California and other parts of the US are similar, even with different ranges of apparent temperature. They both found approximately a $2 \%$ increase in mortality associated with a $10^{\circ} \mathrm{F}$ increase in apparent temperature.

\section{Air Pollutants as Confounders/Effect Modifiers}

Table 2 includes recent studies that have evaluated air pollutants as a potential confounder and/or effect modifier of the high ambient temperature and mortality association. The pollutants that have been examined include ozone $\left(\mathrm{O}_{3}\right)$, particulate matter less than $10 \mu \mathrm{g} / \mathrm{m} 3$ in aerodynamic diameter $\left(\mathrm{PM}_{10}\right)$, fine particulate matter $\left(\mathrm{PM}_{2.5}\right)$, carbon monoxide $(\mathrm{CO})$, sulfur dioxide $\left(\mathrm{SO}_{2}\right)$, and nitrogen dioxide $\left(\mathrm{NO}_{2}\right)$. Most investigators who considered pollutants evaluated $\mathrm{PM}$ and $\mathrm{O}_{3}$, since these pollutants have been found to be associated with mortality and are often correlated with high temperature.
Although the effect estimates changed with pollutants in the model, no significant confounding [15] or effect modification by pollution on the association between temperature and mortality was reported in some recent studies conducted in the US [6,7]. The studies conducted by Bell et al. and Zanobetti and Schwartz considered $\mathrm{PM}_{10}$ (as well as $\mathrm{PM}_{2.5}$ in the Zanobetti and Schwartz study) and $\mathrm{O}_{3}$, while the study by Basu et al. considered $\mathrm{O}_{3}, \mathrm{PM}_{2.5}$, $\mathrm{PM}_{10}, \mathrm{NO}_{2}, \mathrm{CO}$, and $\mathrm{SO}_{2}$. Stafoggia [16] and Rainham and Smoyer-Tomic [17] also reported no confounding by $\mathrm{O}_{3}$ in Italy and Canada, respectively, and Pattenden did not find confounding by markers of PM in both Sofia (total suspended in particulates) and London (black smoke). However, $\mathrm{PM}_{10}$ was found to be confounder in Monterrey, Mexico [18], Sydney, Australia [19], and in regions throughout the United States, especially in the summer [5]. Ren and Tong [20] also observed $\mathrm{PM}_{10}$ to modify the association in their study conducted in Brisbane, Australia. $\mathrm{O}_{3}$ was found to be a confounder especially on hot days $[18,21]$, and other investigators also showed $\mathrm{O}_{3}$ to be a positive effect modifier of temperature and mortality, at least in some study locations $[22,23]$.

The results for confounding and/or effect modification by air pollutants on the temperature-mortality association remain mixed; as stated, some investigators reported air pollutants as confounders or effect modifiers while others found no significant confounding or effect modification in their studies.

\section{Cause-specific Outcomes and Vulnerable Subgroups}

Much of the focus of epidemiologic studies has been identifying cause-specific outcomes and vulnerable subgroups of mortality from high ambient temperature (Table 3 ).

\section{Cause-specific outcomes}

Some investigators have reported greater risks for deaths from cardiovascular (CVD) $[5,14,24]$, respiratory $[5,12,24-26]$, cerebrovascular $[16]$, diabetes $[27,28]$, or pre-existing psychiatric disorders $[16,29,30]$. Other studies also showed elevated risk from mortality subcategories of CVD diseases, such as myocardial infarction $[8,14,31]$, ischemic heart disease [14], and congestive heart failure $[14,30,32]$,

\section{Age}

Age has been found to modify the association between ambient temperature and mortality. The elderly have been reported to be at greater risk from mortality following heat waves, as well as ambient temperature. In addition to the elderly who were at least 75 years [12], 70 years [31] or 65 years $[5,14,15,19,24-26,33,34]$ of age, children under 15 years $[18,33]$, children five years and younger [14], and infants one year of age and under $[14,35]$ have been iden- 
Table I: Recent Studies of High Ambient Temperature and All-Cause Daily Mortality*

\begin{tabular}{|c|c|c|c|c|}
\hline Reference & Study population & Method & Exposure & Result: effect estimate $(95 \% \mathrm{Cl})$ \\
\hline Baccini 2008 [12] & $\begin{array}{l}15 \text { European cities, April-September } \\
1990-2000 \text { ( } 5 \text { - I I years depending on } \\
\text { data availability for city) }\end{array}$ & $\begin{array}{l}\text { Time-series; } \\
\% \text { change }\end{array}$ & $\begin{array}{l}\text { Maximum apparent temperature } \\
\text { (threshold } 29.4^{\circ} \mathrm{C} \text { Mediterranean } \\
\text { cities and } 23.3^{\circ} \mathrm{C} \text { north-continental } \\
\text { cities) }\end{array}$ & $\begin{array}{l}1{ }^{\circ} \mathrm{C} \text { increase above threshold } 3.12(0.60-5.72) \text { in } \\
\text { Mediterranean and } 1.84(0.06-3.64) \text { in north- } \\
\text { continental region } \\
\text { Lag: } 3 \text { days prior }\end{array}$ \\
\hline Basu 2008 [6] & $\begin{array}{l}9 \text { California counties, May to } \\
\text { September 1999-2003 }\end{array}$ & $\begin{array}{l}\text { Time-series and case-crossover; } \\
\% \text { change }\end{array}$ & $\begin{array}{l}\text { Daily apparent temperature } \\
\text { (minimum, mean, maximum); daily } \\
\text { mean } \mathrm{O}_{3}, \mathrm{PM}_{2.5}, \mathrm{PM}_{10}, \mathrm{NO}_{2}, \mathrm{CO} \\
\mathrm{SO}_{2}\end{array}$ & $\begin{array}{l}\text { Per } 10^{\circ} \mathrm{F} \text { increase mean temperature, } 2.3(1.0- \\
3.6) \text {, similar results for minimum and maximum } \\
\text { temperatures } \\
\text { Lag: } 0\end{array}$ \\
\hline Bell 2008 [15] & $\begin{array}{l}\text { Sao Paulo, Brazil, Santiago, Chile and } \\
\text { Mexico City, Mexico, 1998-2002 }\end{array}$ & Case-crossover; \% change & $\begin{array}{l}\text { Same day apparent temperature } \\
\text { compared with days at } 75^{\text {th }} \\
\text { percentile, } \mathrm{O}_{3}, \mathrm{PM}_{10}\end{array}$ & $\begin{array}{l}2.69(-2.06,7.88) \text { for Santiago, } 6.51 \%(3.57,9.52) \\
\text { for Sao Paulo and } 3.22 \%(0.93,5.57) \text { for Mexico } \\
\text { City } \\
\text { Lag: } 0\end{array}$ \\
\hline McMichael 2008 [45] & $\begin{array}{l}\text { Delhi, Monterrey, Mexico City, } \\
\text { Chiang Mai, Bangkok, Salvador, Sao } \\
\text { Paulo, Santiago, Cape Town, } \\
\text { Ljubljana, Bucharest, Sofia, } 2 \text { to 5- } \\
\text { year series } \\
\text { (199I-1999) }\end{array}$ & Time-series; \% change & $\begin{array}{l}\text { Daily maximum threshold }\left(16^{\circ} \mathrm{C} \text { - }\right. \\
\left.31^{\circ} \mathrm{C}\right) \text { temperature, relative } \\
\text { humidity, precipitation data, } \mathrm{PM}_{10} \text {, } \\
\mathrm{BS} \text {, or TSP }\end{array}$ & $\begin{array}{l}1^{\circ} \mathrm{C} \text { increase above threshold increased death } \\
\text { rates with increasing heat in all cities: (ranging } \\
\text { from } 0.77-18.8) \text { except Chiang Mai } 2.39(-0.49- \\
5.35) \text { and Cape Town } 0.47 \\
(-0.31-1.24) \\
\text { Lag: } 2 \text {-day average }\end{array}$ \\
\hline Vaneckova 2008a [46] & $\begin{array}{l}\text { Sydney, Australia, October to March } \\
\text { |993-200I }\end{array}$ & $\begin{array}{l}\text { Time-series; ratio of highest } \\
10 \% \text { mortality days within air } \\
\text { mass and } \% \text { frequency of air } \\
\text { mass occurrence }\end{array}$ & Temporal Synoptic Index (TSI) & $\begin{array}{l}\mathrm{I} .64 \text { and } 2.64 \text { (both significant)for warmest TSIs, } \\
\text { no } \mathrm{Cl} \text { provided }\end{array}$ \\
\hline $\begin{array}{l}\text { Zanobetti and Schwartz } \\
2008[7]\end{array}$ & $\begin{array}{l}9 \text { U.S. counties, May to September } \\
1999-2002\end{array}$ & $\begin{array}{l}\text { Time-series and case-crossover; } \\
\% \text { change }\end{array}$ & $\begin{array}{l}\text { Daily apparent temperature } \\
\text { (minimum, mean, maximum); daily } \\
\text { mean } \mathrm{O}_{3}, \mathrm{PM}_{2.5}, \mathrm{PM}_{10}\end{array}$ & $\begin{array}{l}\text { Per } 10^{\circ} \mathrm{F} \text { increase mean temperature, } 1.8(1.09 \text { - } \\
2.5) \text { case-crossover and } 2.7(2.0-3.5) \text { time-series; } \\
\text { similar results for minimum and maximum } \\
\text { temperatures } \\
\text { Lag: } 0\end{array}$ \\
\hline Barnett 2007 [47] & $\begin{array}{l}\text { I07 U.S cities using data from the } \\
\text { National Morbidity and Mortality } \\
\text { Study, } 1987-2000\end{array}$ & $\begin{array}{l}\text { Case-crossover; } \\
\% \text { change }\end{array}$ & Daily temperature & $\begin{array}{l}\text { Per } 10^{\circ} \mathrm{F} \text {, summer } 1987 \text { average increase in } \\
\text { cardiovascular deaths was } 4.7(3.0-6.5) \text {. By } \\
\text { summer } 2000 \text {, the risk with higher temperature } \\
\text { had disappeared }(-0.4,-3.2-2.5) \\
\text { Lag: } 04\end{array}$ \\
\hline Medina-Ramon 2007 [2I] & $\begin{array}{l}50 \text { US cities in cold (November to } \\
\text { March) and warm (May to } \\
\text { September) seasons }\end{array}$ & $\begin{array}{l}\text { Case-crossover; } \\
\% \text { change }\end{array}$ & $\begin{array}{l}\text { Binary variable as extreme heat } \\
\text { (range } 22-32^{\circ} \mathrm{C} \text { ) } \\
\text { and continuous; } \mathrm{O}_{3}\end{array}$ & $\begin{array}{l}5.74(3.38-8.15) \text { for extreme heat } \\
\text { Lag: 2-day average }\end{array}$ \\
\hline Kolb 2007 [32] & Montreal, Canada 1984-1993 & $\begin{array}{l}\text { Case-crossover; } \\
\text { odds ratio }\end{array}$ & $\begin{array}{l}\text { Mean daily and maximum } \\
\text { temperature, barometric pressure, } \\
\text { relative humidity, adjusted for } \mathrm{O}_{3} \\
\text { and both } \mathrm{NO}_{2} \text { and } \mathrm{O}_{3}\end{array}$ & $\begin{array}{l}1.20(1.14-1.38) \text { for } 25-30^{\circ} \mathrm{C} \text { maximum } \\
\text { temperature; strong nonlinear association with a } \\
\text { threshold at } 25^{\circ} \mathrm{C} \\
\text { Lag: average } 02 \text {; no association after } 3 \text { days }\end{array}$ \\
\hline
\end{tabular}

107 U.S cities using data from the Case-crossover
National Morbidity and Mortality $\%$ change National Morbidity threshold at $25^{\circ} \mathrm{C}$

Lag: average 02 ; no association after 3 days 


\begin{tabular}{|c|c|c|c|c|}
\hline Carson 2006 [48] & $\begin{array}{l}\text { London, England, } \\
4 \text { time periods, winter: December- } \\
\text { March; non-winter: April-November }\end{array}$ & $\begin{array}{l}\text { Time-series; ratio of winter to } \\
\text { non-winter deaths }\end{array}$ & Daily mean temperature & $\begin{array}{l}1.24(1.16-1.34) \text { from } 1900-10, ; 1.54(1.42,1.68) \\
\text { from } 1927-37,1.48(1.35,-1.64) \text { from } 1954-64, \\
1.22(1.13-1.31) \text { from 1986-96; heat deaths } \\
\text { diminished overall in the century }\end{array}$ \\
\hline Kim 2006 [40] & $\begin{array}{l}6 \text { cities in South Korea, summer } \\
1994-2006\end{array}$ & $\begin{array}{l}\text { Time-series; } \\
\% \text { change }\end{array}$ & $\begin{array}{l}\text { Daily mean temperature thresholds } \\
\left(27-29.7^{\circ} \mathrm{C}\right)\end{array}$ & $\begin{array}{l}1{ }^{\circ} \mathrm{C} \text { above threshold } 16.3(14.2,18.4), 9.10 \\
(5.12,13.2), 7.01(4.42,9.66), 6.73(2.47,11.2) \\
\text { for Seoul, Daegu, Incheon and Gwangiu, } \\
\text { respectively }\end{array}$ \\
\hline Michelozzi 2006 [49] & $\begin{array}{l}4 \text { Italian cities, June to September } \\
2003 \text { \& } 2004 \text { and reference period } \\
\text { (Roma, Torino, Milano: 1995-2002 } \\
\text { and Bologna: } \\
\text { 1996-2002) }\end{array}$ & $\begin{array}{l}\text { Time-series; } \\
\% \text { change }\end{array}$ & $\begin{array}{l}\text { Daily maximum apparent } \\
\text { temperature thresholds }\left(28-32^{\circ} \mathrm{C}\right)\end{array}$ & $\begin{array}{l}1{ }^{\circ} \mathrm{C} \text { above threshold } 3.2 \text { (1.9-4.6), } 5.0 \text { (3.8-6.I), } \\
5.4(4.3-6.5), 3.8(2.5-5.0) \text { for Bologna, Milano, } \\
\text { Roma, and Torino, respectively }\end{array}$ \\
\hline Stafoggia 2006 [16] & $\begin{array}{l}\text { Bologna, Milan, Rome, Turin, 1997- } \\
2003\end{array}$ & Case-crossover; odds ratio & $\begin{array}{l}30^{\circ} \mathrm{C} \text { mean apparent temperature } \\
\text { relative to } 20^{\circ} \mathrm{C} \text {; odds ratio }\end{array}$ & $\begin{array}{l}\text { I.34 }(1.27,1.42) \\
\text { Lag: } 01\end{array}$ \\
\hline Basu 2005 [5] & $\begin{array}{l}20 \text { US metropolitan areas, seasonal } \\
\text { analysis } 1992\end{array}$ & $\begin{array}{l}\text { Time series (relative risk) and } \\
\text { case-crossover } \\
\text { (odds ratio) }\end{array}$ & $\begin{array}{l}\text { Mean daily temperature per IOF } \\
\text { adjusted for dew point temperature; } \\
\text { daily } \mathrm{O}_{3}\end{array}$ & $\begin{array}{l}\text { Per } 10^{\circ} \mathrm{F}, \mathrm{I} .15(\mathrm{I} .07-1.24), \mathrm{I} .10(0.96-1.27), 1.08 \\
(0.92-1.26), 1.08(\mathrm{I} .02-1.15) \text {, and I.0I }(0.92-1.1 \mathrm{I}) \\
\text { in the Southwest, Southeast, Northwest, } \\
\text { Northeast, and Midwest, respectively, in the } \\
\text { summer from the time-stratified case-crossover } \\
\text { Lag: } 0,1\end{array}$ \\
\hline El-Zein 2004 [34] & Greater Beirut, Lebanon, 1997-1999 & $\begin{array}{l}\text { Time-series; } \\
\% \text { change }\end{array}$ & $\begin{array}{l}\text { Mean daily temperature, mean daily } \\
\text { humidity, minimum mortality } \\
\text { temperature }(\mathrm{TMM})=27.5^{\circ} \mathrm{C}\end{array}$ & $\begin{array}{l}1^{\circ} \mathrm{C} \text { above TMM I } 2.3(5.7,19.4 \%) \text { increase in } \\
\text { annual mortality } \\
\text { Lag: } 0\end{array}$ \\
\hline Goodman 2004 [26] & $\begin{array}{l}\text { Dublin, Ireland, April } 1980 \text { to } \\
\text { December } 1996\end{array}$ & $\begin{array}{l}\text { Time-series; } \\
\% \text { change }\end{array}$ & $\begin{array}{l}\text { Daily minimum temperature, daily } \\
\text { mean relative humidity }\end{array}$ & $\begin{array}{l}1^{\circ} \mathrm{C} \text { increase } 0.4(0.3-0.6) \text { increase } \\
\text { Lag: } 0\end{array}$ \\
\hline Pattenden 2003 [50] & $\begin{array}{l}\text { Sofia, Bulgaria (1996-1999) and } \\
\text { London, England (1993-1996) }\end{array}$ & $\begin{array}{l}\text { Time-series; } \\
\% \text { change }\end{array}$ & $\begin{array}{l}\text { Daily mean temperature, relative } \\
\text { humidity and PM (black smoke for } \\
\text { London and total suspended } \\
\text { particulates for Sofia) }\end{array}$ & $\begin{array}{l}1^{\circ} \mathrm{C} \text { increase above } 90 \text { th } \% 1.9(1.4 \text { to } 2.4) \text { in } \\
\text { London, and } 3.5(2.2 \text { to } 4.8) \text { in Sofia } \\
\text { Lag: } 2 \text { day average }\end{array}$ \\
\hline Curriero 2002 [39] & II Eastern US cities, 1973-1994 & $\begin{array}{l}\text { Time-series; } \\
\% \text { change }\end{array}$ & $\begin{array}{l}\text { Daily mean temperature, dew point } \\
\text { temperature; minimum mortality } \\
\text { temperature (MMT) range: } 65.2-90.3\end{array}$ & $\begin{array}{l}\text { Per } 10^{\circ} \mathrm{F} \text { above } M M T \text { range } 1.4-6.7 \\
\text { Lag: } 0\end{array}$ \\
\hline Braga 200I [lI] & 12 US cities. 1986-1993 & $\begin{array}{l}\text { Time-series; } \\
\% \text { increase }\end{array}$ & $\begin{array}{l}\text { Mean daily temperature, relative } \\
\text { humidity }\end{array}$ & $\begin{array}{l}\text { 4\% increase (no } \mathrm{Cl} \text { given); } \\
\text { Lag: } 0 \text { or I } \\
\text { Harvesting effect for hot temperatures }\end{array}$ \\
\hline
\end{tabular}

* Exceptions: El-Zein 2004 and Carson 2006 reported annual and weekly deaths, respectively. 
Table 2: Recent Studies of High Ambient Temperature and Mortality Examining Air Pollutants as Potential Confounders and/or Effect Modifiers

\begin{tabular}{|c|c|c|c|c|c|}
\hline Reference & Study location & Method & Exposure & Causes of death & Result \\
\hline Basu 2008 [6] & $\begin{array}{l}9 \text { California counties, } \\
\text { May to September } \\
1999-2003\end{array}$ & $\begin{array}{l}\text { Time-series and case- } \\
\text { crossover }\end{array}$ & $\begin{array}{l}\text { Same day mean } \\
\text { apparent } \\
\text { temperature; daily } \\
\text { mean } \mathrm{O}_{3} \text {, also } \mathrm{PM}_{2.5} \text {, } \\
\mathrm{PM}_{10}, \mathrm{NO}_{2}, \mathrm{CO}, \mathrm{SO}_{2} \text {, } \\
\text { lag } 0 \text { for } \mathrm{PM} \text {, lagol for } \\
\text { gases }\end{array}$ & All-cause mortality & $\begin{array}{l}\text { Confounders: none } \\
\text { found } \\
\text { Effect modifiers: none } \\
\text { found }\end{array}$ \\
\hline Bell 2008 [15] & $\begin{array}{l}\text { Sao Paulo, Brazil, } \\
\text { Santiago, Chile and } \\
\text { Mexico City, Mexico, } \\
\text { 1998-2002 }\end{array}$ & Case-crossover & $\begin{array}{l}\text { Same day apparent } \\
\text { temperature } \\
\text { compared with days at } \\
75^{\text {th }} \text { percentile, same } \\
\text { day lag } \mathrm{O}_{3} \text {, same-day } \\
\text { lag } \mathrm{PM}_{10} \text { except } \\
\text { Santiago lag I PM } \\
10\end{array}$ & $\begin{array}{l}\text { All-cause daily } \\
\text { mortality }\end{array}$ & $\begin{array}{l}\text { Confounders: } \mathrm{O}_{3} \text {, } \\
\mathrm{PM}_{10} \\
\text { Effect modifiers: not } \\
\text { studied }\end{array}$ \\
\hline McMichael 2008 [45] & $\begin{array}{l}\text { Delhi, Monterrey, } \\
\text { Mexico City, Chiang } \\
\text { Mai, Bangkok, } \\
\text { Salvador, Sao Paulo, } \\
\text { Santiago, Cape Town, } \\
\text { Ljubljana, Bucharest, } \\
\text { Sofia, } 2 \text { to 5-year } \\
\text { series (1991-1999) }\end{array}$ & Time-series & $\begin{array}{l}\text { Daily maximum } \\
\text { threshold }\left(16^{\circ} \mathrm{C} \text { - }\right. \\
\left.31{ }^{\circ} \mathrm{C}\right) \text { temperature, } \\
\text { relative humidity, } \\
\text { precipitation data, } \\
\mathrm{PM}_{10}, \mathrm{BS} \text {, or TSP }\end{array}$ & All-cause mortality & $\begin{array}{l}\text { Confounders: none } \\
\text { found } \\
\text { Effect modifiers: not } \\
\text { studied }\end{array}$ \\
\hline Ren 2008 [22] & $\begin{array}{l}\text { US } 95 \text { NMMAPS } \\
\text { counties, June to } \\
\text { September } 1987-2000\end{array}$ & Time-series & $\begin{array}{l}\text { Daily maximum } \\
\text { temperature (same- } \\
\text { day, lag } \mathrm{I} \text { ), maximum } \\
\text { hourly } \mathrm{O}_{3}\end{array}$ & CVD mortality & $\begin{array}{l}\text { Confounders: not } \\
\text { studied } \\
\text { Effect modifier: } \mathrm{O}_{3}\end{array}$ \\
\hline Vaneckova 2008a [46] & $\begin{array}{l}\text { Sydney, Australia, } \\
\text { October to March } \\
\text { 1993-200I }\end{array}$ & Time-series & $\begin{array}{l}\text { Temporal Synoptic } \\
\text { Index (TSI) on the } \\
\text { highest } 10 \% \text { mortality } \\
\text { days, } \mathrm{O}_{3}, \mathrm{PM}_{10}\end{array}$ & $\begin{array}{l}\text { All-cause, circulatory, } \\
\text { cerebrovascular }\end{array}$ & $\begin{array}{l}\text { Confounders: } \mathrm{O}_{3} \text { on } \\
\text { warm, humid days and } \\
\mathrm{PM}_{10} \text { on hot, dry days } \\
\text { Effect modifiers: not } \\
\text { studied }\end{array}$ \\
\hline Vaneckova 2008b [19] & $\begin{array}{l}\text { Sydney, Australia, } \\
\text { October to March } \\
\text { 1993-2004 }\end{array}$ & Time-series & $\begin{array}{l}\text { Daily maximum } \\
\text { temperature, } \\
\text { maximum } \mathrm{O}_{3}\end{array}$ & $\begin{array}{l}\text { Underlying and } \\
\text { associated causes of } \\
\text { death }\end{array}$ & $\begin{array}{l}\text { Confounders: } \mathrm{O}_{3} \text {, } \\
\mathrm{PM}_{10} \\
\text { Effect modifiers: not } \\
\text { studied }\end{array}$ \\
\hline $\begin{array}{l}\text { Zanobetti and } \\
\text { Schwartz } 2008 \text { [7] }\end{array}$ & $\begin{array}{l}9 \text { U.S. counties, May } \\
\text { to September 1999- } \\
2002\end{array}$ & $\begin{array}{l}\text { Time series and case- } \\
\text { crossover }\end{array}$ & $\begin{array}{l}\text { Daily apparent } \\
\text { temperature } \\
\text { (minimum, mean, } \\
\text { maximum); daily mean } \\
\mathrm{O}_{3}, \mathrm{PM}_{2.5}\end{array}$ & All-cause mortality & $\begin{array}{l}\text { Confounders: none } \\
\text { found } \\
\text { Effect modifiers: none } \\
\text { found }\end{array}$ \\
\hline Kolb 2007 [32] & $\begin{array}{l}\text { Montreal, Canada } \\
\text { 1984-1993 }\end{array}$ & Case-crossover & $\begin{array}{l}\text { Mean daily and } \\
\text { maximum } \\
\text { temperature, } \\
\text { barometric pressure, } \\
\text { relative humidity, } \\
\text { adjusted for } \mathrm{O}_{3} \text { and } \\
\text { both } \mathrm{NO}_{2} \text { and } \mathrm{O}_{3}\end{array}$ & $\begin{array}{l}\text { Daily all-cause } \\
\text { mortality }\end{array}$ & $\begin{array}{l}\text { Confounders: none } \\
\text { found } \\
\text { Effect modifiers: not } \\
\text { studied }\end{array}$ \\
\hline $\begin{array}{l}\text { Medina-Ramon } 2007 \\
{[21]}\end{array}$ & $\begin{array}{l}50 \text { US cities in cold } \\
\text { (November to March) } \\
\text { and warm (May to } \\
\text { September) seasons }\end{array}$ & Case-crossover & $\begin{array}{l}\text { Binary variable as } \\
\text { extreme temperature } \\
\text { and continuous; } \mathrm{O}_{3}\end{array}$ & $\begin{array}{l}\text { All-cause and CVD } \\
\text { mortality }\end{array}$ & $\begin{array}{l}\text { Confounder: } \mathrm{O}_{3} \\
\text { Effect modifiers: not } \\
\text { studied }\end{array}$ \\
\hline
\end{tabular}


Table 2: Recent Studies of High Ambient Temperature and Mortality Examining Air Pollutants as Potential Confounders and/or Effect Modifiers (Continued)

\begin{tabular}{|c|c|c|c|c|c|}
\hline Filleul 2006 [23] & $\begin{array}{l}9 \text { French cities, all } \\
\text { year and heat wave } \\
\text { August } 2003\end{array}$ & Time-series & $\begin{array}{l}\text { Minimum and } \\
\text { maximum } \\
\text { temperature, 8-hour } \\
\text { maximum } \mathrm{O}_{3}\end{array}$ & $\begin{array}{l}\text { Daily all-cause } \\
\text { mortality }\end{array}$ & $\begin{array}{l}\text { Confounders: not } \\
\text { studied } \\
\text { Effect modifier: } \mathrm{O}_{3} \text { for } \\
\text { some cities }\end{array}$ \\
\hline Ren 2006 [5I] & $\begin{array}{l}\text { Brisbane, Australia (all } \\
\text { year January } 1996 \text { to } \\
\text { December 200I) }\end{array}$ & Time-series & $\begin{array}{l}\text { Minimum } \\
\text { temperature, daily } \\
\mathrm{PM}_{10} \text { as modifier }\end{array}$ & $\begin{array}{l}\text { Cardiorespiratory } \\
\text { mortality }\end{array}$ & $\begin{array}{l}\text { Confounders: not } \\
\text { studied } \\
\text { Effect modifier: } \mathrm{PM}_{10}\end{array}$ \\
\hline Stafoggia 2006 [16] & $\begin{array}{l}\text { Bologna, Milan, Rome, } \\
\text { Turin, 1997-2003 }\end{array}$ & Case-crossover & $\begin{array}{l}30^{\circ} \mathrm{C} \text { mean apparent } \\
\text { temperature (lag0 } \mathrm{I}) \\
\text { relative to } 20^{\circ} \mathrm{C}, \mathrm{O}_{3}\end{array}$ & All-cause mortality & $\begin{array}{l}\text { Confounders: none } \\
\text { found } \\
\text { Effect modifiers: not } \\
\text { studied }\end{array}$ \\
\hline Basu 2005 [5] & $\begin{array}{l}20 \text { US metropolitan } \\
\text { areas, seasonal } \\
\text { analysis } 1992\end{array}$ & $\begin{array}{l}\text { Time series (and case- } \\
\text { crossover }\end{array}$ & $\begin{array}{l}\text { Mean daily } \\
\text { temperature per } 10^{\circ} \mathrm{F} \\
\text { adjusted for dew } \\
\text { point temperature; } \\
\text { daily } \mathrm{O}_{3}\end{array}$ & $\begin{array}{l}\text { Individual and daily } \\
\text { cardiorespiratory } \\
\text { mortality }\end{array}$ & $\begin{array}{l}\text { Confounders: } \mathrm{PM}_{10} \text { in } \\
\text { summer } \\
\text { Effect modifiers: not } \\
\text { studied }\end{array}$ \\
\hline O'Neill 2005 [18] & $\begin{array}{l}\text { Mexico City (1996- } \\
98) \text { and Monterrey } \\
(1996-99)\end{array}$ & $\begin{array}{l}\text { Time series; } \\
\% \text { change }\end{array}$ & $\begin{array}{l}\text { Heat }\left(35-36^{\circ} \mathrm{C} \text { for }\right. \\
\text { Monterrey), mean } \\
\text { temperature }\left(25^{\circ} \mathrm{C}\right. \\
\text { Monterrey, } 15^{\circ} \mathrm{C} \\
\text { Mexico City), daily } \mathrm{O}_{3}\end{array}$ & $\begin{array}{l}\text { Daily all-cause } \\
\text { mortality }\end{array}$ & $\begin{array}{l}\text { Confounders: } \mathrm{O}_{3} \text { and } \\
\mathrm{PM}_{10} \text { on hot days } \\
\text { Effect modifiers: not } \\
\text { studied }\end{array}$ \\
\hline $\begin{array}{l}\text { Rainham and Smoyer- } \\
\text { Tomic } 2003 \text { [17] }\end{array}$ & $\begin{array}{l}\text { Toronto, May I to } \\
\text { September 30, } 1980- \\
1996\end{array}$ & $\begin{array}{l}\text { Time-series; relative } \\
\text { risk (RR) }\end{array}$ & $\begin{array}{l}\text { Humidex, } \mathrm{O}_{3} \text {, also } \\
\mathrm{CO}, \mathrm{NO}_{2}, \mathrm{SO}_{2}\end{array}$ & $\begin{array}{l}\text { Daily all-cause } \\
\text { mortality }\end{array}$ & $\begin{array}{l}\text { Confounders: none } \\
\text { found } \\
\text { Effect modifiers: not } \\
\text { studied }\end{array}$ \\
\hline Pattenden 2003 [49] & $\begin{array}{l}\text { Sofia, Bulgaria (1996- } \\
\text { 1999) and London, } \\
\text { England (1993-1996) }\end{array}$ & $\begin{array}{l}\text { Time-series; } \\
\% \text { change }\end{array}$ & $\begin{array}{l}\text { Daily weather (2-day } \\
\text { mean) and PM (black } \\
\text { smoke for London } \\
\text { and total suspended } \\
\text { particulates for Sofia) }\end{array}$ & $\begin{array}{l}\text { Daily all-cause } \\
\text { mortality }\end{array}$ & $\begin{array}{l}\text { Confounders: none } \\
\text { found } \\
\text { Effect modifiers: not } \\
\text { studied }\end{array}$ \\
\hline
\end{tabular}

tified to be at increased risk for mortality from high ambient temperature. One investigator also reported 15 to 64 years of age to be at a significantly increased risk, although still lower than the elderly or young children [33].

\section{Gender}

Modifications by gender has also been studied, and some investigators reported no difference by gender [14], while others found men in Santiago and Sao Paulo [15] specifically for circulatory causes [35] or women in various locations $[15-17,19,24,36]$ to be at higher risk for mortality.

\section{Racelethnic group}

Other recent epidemiologic studies also reported Black racial ethnic group [14,37] and non-Whites [28] to be at greater risk than Whites in the US. Hispanic subgroups, however, have not been identified as being at greater risk in one study, partially explained by more social networking among this ethnic group [14].

\section{Socioeconomic factors}

Other factors that provoked greater risk included indicators for lower socioeconomic status, including the less educated, persons living in lower income areas [16] and dying out of the hospital $[14,27,38]$. Also, increased poverty [39], and lack of air conditioner [11,37,39] were observed risk factors. However, lower socioeconomic status [33] and education level were not found to be a risk factor in all studies [14].

\section{Latitude Variations}

Some studies reported variation by latitude, supporting the evidence for acclimatization. People who live in cities where the temperatures are generally elevated in the summer were found to have higher minimum mortality temperatures, or less risk given the same level of temperature, than people who live in cities with milder climates $[7,21,39]$. A similar finding was reported in California, where slightly higher estimates were found for coastal 
Table 3: Recent Studies Identifying Vulnerable Subgroups of Mortality from High Ambient Temperature

\begin{tabular}{|c|c|c|c|c|c|}
\hline Reference & Study location & Study design & Exposure & Causes of death & Result \\
\hline Baccini 2008 [12] & $\begin{array}{l}\text { I5 European cities, } \\
\text { April-September 1990- } \\
2000 \text { (5-II years } \\
\text { depending on data } \\
\text { availability for city) }\end{array}$ & Time-series & $\begin{array}{l}\text { Maximum apparent } \\
\text { temperature (threshold } \\
29.4^{\circ} \mathrm{C} \text { Mediterranean } \\
\text { cities and } 23.3^{\circ} \mathrm{C} \text { north- } \\
\text { continental cities) }\end{array}$ & $\begin{array}{l}\text { Daily all-cause } \\
\text { mortality }\end{array}$ & $\begin{array}{l}\text { Respiratory diseases } \\
\text { among } 75+\text { years }\end{array}$ \\
\hline $\begin{array}{l}\text { Basu and Ostro } \\
2008[14]\end{array}$ & $\begin{array}{l}9 \text { California counties, } \\
\text { May to September } \\
\text { 1999-2003 }\end{array}$ & Case-crossover & $\begin{array}{l}\text { Mean daily apparent } \\
\text { temperature }\end{array}$ & $\begin{array}{l}\text { Cause-specific } \\
\text { mortality; all-cause } \\
\text { mortality by age, race/ } \\
\text { ethnicity, gender, } \\
\text { education level }\end{array}$ & $\begin{array}{l}\text { Cardiovascular, higher } \\
\text { specifically for ischemic } \\
\text { heart disease, myocardial } \\
\text { infarction, and congestive } \\
\text { heart failure, } \leq \text { I year, } \leq 5 \\
\text { years, elderly, Black race, } \\
\text { out of hospital death; no } \\
\text { elevated risks for } \\
\text { cerebrovascular, diabetes, } \\
\text { respiratory; no difference } \\
\text { by gender or high school } \\
\text { graduation }\end{array}$ \\
\hline Bell 2008 [15] & $\begin{array}{l}\text { Sao Paulo, Brazil, } \\
\text { Santiago, Chile and } \\
\text { Mexico City, Mexico, } \\
\text { 1998-2002 }\end{array}$ & Case-crossover & $\begin{array}{l}\text { Same day apparent } \\
\text { temperature }\end{array}$ & $\begin{array}{l}\text { Daily all-cause } \\
\text { mortality }\end{array}$ & $\begin{array}{l}65+\text { years, women in } \\
\text { Mexico City, but men in } \\
\text { Santiago and Sao Paulo, } \\
\text { less educated in Sao Paulo }\end{array}$ \\
\hline Ishigami 2008 [24] & $\begin{array}{l}\text { Budapest, London and } \\
\text { Milan, } 2003\end{array}$ & Time-series & $\begin{array}{l}\text { Mean daily temperature } \\
\text { (lag0 and lagI), PM }{ }_{10} \text { (TSP } \\
\text { in Budapest), ozone }\end{array}$ & $\begin{array}{l}\text { Daily all-cause } \\
\text { mortality }\end{array}$ & $\begin{array}{l}\text { Increased age, females } \\
65+\text { years greater risk in } \\
\text { London and Milan and } \\
\text { non-elderly adults in } \\
\text { Milan; mortality from } \\
\text { external causes, } \\
\text { respiratory and } \\
\text { cardiovascular diseases }\end{array}$ \\
\hline Stafoggia 2008 [30] & $\begin{array}{l}4 \text { Italian cities, } 1997- \\
2004\end{array}$ & Case-crossover & $\begin{array}{l}\text { Apparent temperature } \\
30^{\circ} \mathrm{C} \text { compared to } 20^{\circ} \mathrm{C}\end{array}$ & $\begin{array}{l}\text { Deaths in hospitals for } \\
\text { those with } 2+\text { days in } \\
\text { hospital }\end{array}$ & $\begin{array}{l}\text { Increased age, single } \\
\text { general medicine } \\
\text { compared to high and } \\
\text { intensive care units, } \\
\text { history of psychiatric } \\
\text { disorders, } \\
\text { cerebrovascular diseases, } \\
\text { heart failure, stroke, } \\
\text { chronic pulmonary } \\
\text { diseases }\end{array}$ \\
\hline $\begin{array}{l}\text { Vaneckova 2008a } \\
\text { [46] }\end{array}$ & $\begin{array}{l}\text { Sydney, Australia, } \\
\text { October to March } \\
\text { |993-200| }\end{array}$ & Time-series & $\begin{array}{l}\text { Temporal Synoptic Index } \\
\text { (TSI); ratio of highest I0\% } \\
\text { mortality days within air } \\
\text { mass and \% frequency of } \\
\text { air mass occurrence }\end{array}$ & $\begin{array}{l}\text { Daily all-cause } \\
\text { mortality }\end{array}$ & $65+$ years, women \\
\hline Yip 2008 [52] & $\begin{array}{l}\text { Maricopa County, } \\
\text { Arizona, June to } \\
\text { September } \\
2000-2005\end{array}$ & Time-series & Heat index & Heat-related deaths & $\begin{array}{l}\text { Young and old outdoors, } \\
\text { but greater risk for elderly } \\
\text { indoors }\end{array}$ \\
\hline Hajat 2007 [25] & $\begin{array}{l}\text { England and Wales, } \\
\text { 1993-2003 }\end{array}$ & Time-series & 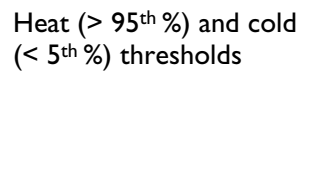 & All-cause mortality & $\begin{array}{l}\text { Elderly, those in nursing } \\
\text { care homes respiratory } \\
\text { and external causes, } \\
\text { women; not modified by } \\
\text { deprivation in London }\end{array}$ \\
\hline
\end{tabular}


Table 3: Recent Studies Identifying Vulnerable Subgroups of Mortality from High Ambient Temperature (Continued)

\begin{tabular}{|c|c|c|c|c|c|}
\hline $\begin{array}{l}\text { Medina-Ramon } \\
2007[2 I]\end{array}$ & $\begin{array}{l}50 \text { US cities in cold } \\
\text { (November to March) } \\
\text { and warm (May to } \\
\text { September) seasons }\end{array}$ & Case-crossover & $\begin{array}{l}\text { Binary variable as } \\
\text { extreme temperature and } \\
\text { continuous; ozone }\end{array}$ & $\begin{array}{l}\text { All-cause and CVD } \\
\text { mortality }\end{array}$ & $\begin{array}{l}\text { Cities with milder } \\
\text { summers, less air } \\
\text { conditioning and higher } \\
\text { population density }\end{array}$ \\
\hline Diaz 2006 [35] & $\begin{array}{l}\text { Madrid, January 1986- } \\
\text { December } 1997\end{array}$ & Time-series & $\begin{array}{l}\mathrm{T}(\text { hwave })=\text { Tmax }-36.5 \mathrm{C} \\
\text { if } \mathrm{Tmax}>36.5 \mathrm{C} ; 5^{\text {th }} \% \text { to } \\
95^{\text {th }} \% \text { temperature, } \mathrm{NO}_{2}\end{array}$ & $\begin{array}{l}A R=(R R-I) / R R \text { for } \\
\text { daily mortality }\end{array}$ & $\begin{array}{l}\text { Circulatory causes, males } \\
45-64 \text { years }\end{array}$ \\
\hline Stafoggia 2006 [16] & $\begin{array}{l}\text { Bologna, Milan, Rome, } \\
\text { Turin, 1997-2003 }\end{array}$ & Case-crossover & $\begin{array}{l}30^{\circ} \mathrm{C} \text { mean apparent } \\
\text { temperature (lag0 }) \\
\text { relative to } 20^{\circ} \mathrm{C} \text {; odds } \\
\text { ratio }\end{array}$ & $\begin{array}{l}\text { All-cause mortality and } \\
\text { previous } \\
\text { hospitalization }\end{array}$ & $\begin{array}{l}\text { Increased age and greater } \\
\text { for women, widows and } \\
\text { widowers, psychiatric } \\
\text { disorders, depression, } \\
\text { heart and circulatory } \\
\text { disorders }\end{array}$ \\
\hline Hajat 2005 [48] & $\begin{array}{l}\text { Delhi, Sao Paulo, } \\
\text { London, January I99I- } \\
\text { December } 1994\end{array}$ & Time-series & $\begin{array}{l}\text { Daily temperature } \\
\text { (lag } 0,1) \text { greater than } \\
20^{\circ} \mathrm{C}\end{array}$ & $\begin{array}{l}\text { Daily all-cause } \\
\text { mortality }\end{array}$ & $\begin{array}{l}\text { Respiratory deaths in Sao } \\
\text { Paulo and London; } \\
\text { children in Delhi }\end{array}$ \\
\hline $\begin{array}{l}\text { O'Neill, Zanobetti } \\
\text { and Schwartz } 2005 \\
\text { [37] }\end{array}$ & $\begin{array}{l}\text { Chicago, Detroit, } \\
\text { Minneapolis, Pittsburgh, } \\
\text { 1988-1993 for Chicago } \\
\text { and 1986-1993 for } \\
\text { other cities }\end{array}$ & Time-series & $\begin{array}{l}\text { Percent change daily } \\
\text { mean temperature } 29^{\circ} \mathrm{C} \\
\text { relative to } 15^{\circ} \mathrm{C} \text { (lag0), } \\
\text { barometric pressure, day } \\
\text { of the week, } \mathrm{PM}_{10}\end{array}$ & $\begin{array}{l}\text { Mortality, prevalence } \\
\text { of air conditioner (AC) }\end{array}$ & $\begin{array}{l}\text { Black race, lack of air } \\
\text { conditioner }\end{array}$ \\
\hline Gouveia 2003 [33] & $\begin{array}{l}\text { Sao Paulo, Brazil, |99|- } \\
\text { I994 }\end{array}$ & Time-series & $\begin{array}{l}\text { Daily mean temperature } \\
\text { (lagOI), } \mathrm{SO}_{2}, \mathrm{PM}_{10}, \mathrm{CO}, \\
\mathrm{O}_{3}, \mathrm{NO}_{2} \text {, day of the } \\
\text { week, season, humidity }\end{array}$ & $\begin{array}{l}\text { Daily all-cause } \\
\text { mortality, excluding } \\
\text { violent deaths, } \\
\text { cardiovascular and } \\
\text { respiratory mortality }\end{array}$ & $\begin{array}{l}\text { Greatest for } 65+\text { years and } \\
<15 \text { years, also increased } \\
\text { for } 15-64 \text { years; elderly } \\
\text { cardiovascular, } \\
\text { respiratory for adults and } \\
\text { elderly; no modification by } \\
\text { socioeconomic status }\end{array}$ \\
\hline O'Neill 2003 [38] & $\begin{array}{l}7 \text { US cities, } \\
1986-1993\end{array}$ & Time-series & $\begin{array}{l}\text { Mean daily apparent } \\
\text { temperature }(\% \text { change } \\
\left.29^{\circ} \mathrm{C} \text { and }-5^{\circ} \mathrm{C}\right), \mathrm{PM}_{10}\end{array}$ & $\begin{array}{l}\text { Daily all-cause } \\
\text { mortality, looking at } \\
\text { effect modification by } \\
\text { demographics \& other } \\
\text { variables }\end{array}$ & $\begin{array}{l}\text { Black race, less educated, } \\
\text { and outside hospital }\end{array}$ \\
\hline $\begin{array}{l}\text { Rainham and } \\
\text { Smoyer-Tomic } \\
2003 \text { [42] }\end{array}$ & $\begin{array}{l}\text { Toronto, May I to } \\
\text { September 30, } 1980- \\
1996\end{array}$ & Time-series & $\begin{array}{l}\text { Humidex, } \mathrm{CO}, \mathrm{O}_{3}, \mathrm{NO}_{2} \text {, } \\
\mathrm{SO}_{2}\end{array}$ & $\begin{array}{l}\text { Daily all-cause } \\
\text { mortality }\end{array}$ & Females \\
\hline Curriero 2002 [39] & $\begin{array}{l}\text { II Eastern US cities, } \\
\text { 1973-1994 }\end{array}$ & Time-series & $\begin{array}{l}\text { Daily mean temperature, } \\
\text { dew point temperature; } \\
\text { minimum mortality } \\
\text { temperature (MMT) } \\
\text { range: } 65.2-90.3\end{array}$ & $\begin{array}{l}\text { Daily all-cause } \\
\text { mortality, excluding } \\
\text { accidents }\end{array}$ & $\begin{array}{l}\text { Higher latitude, more } \\
\text { poverty, less air } \\
\text { conditioning or heating }\end{array}$ \\
\hline
\end{tabular}

counties where milder temperatures are generally experienced [6]. Although coastal areas in California are usually more expensive, many of the homes lack air conditioning, since they have not been needed. Therefore, air conditioning prevalence is not an indicator of socioeconomic status in California, as it is in the remainder of the U.S.

\section{Discussion}

In the past few years, several epidemiologic studies have been conducted in various locations to characterize temperature and mortality. In the US, similar effects were found in nine counties in California and in nine counties outside of California in two separate studies using the same methods [6,7]. In Europe and Korea, however, the effect estimates were larger $[12,40]$, further supporting the need to conduct temperature-mortality studies for specific areas. The results from future studies can be more readily compared if estimates are reported per degree Celsius or Fahrenheit per unit change in temperature (assuming linearity), or if a regression coefficient is given, rather than selecting a threshold value for temperature. In addition, investigators should consider accounting for air pollutants and identifying vulnerable subgroups in their epidemiologic studies. 
The recent epidemiologic evidence suggests that PM and $\mathrm{O}_{3}$ may be confounders, and some studies also found $\mathrm{O}_{3}$ to be an effect modifier in the warmer months. In other words, the association between temperature and mortality is partially a result of the effect of PM and $\mathrm{O}_{3}$. However, this confounding effect is relatively small, and there is clearly an independent effect of both temperature and air pollution on mortality. Others have reported that temperature has a greater effect on mortality with higher levels of $\mathrm{O}_{3}$ (i.e., synergism). Some of the conflicting evidence for confounding and effect modification by air pollutants may be due to high correlations between pollutants and temperature, making it difficult to tease apart the independent effects of either exposure. Also, different sources, chemistry, size distribution of particles, compositions and patterns of exposure [41] of gases and particles are observed throughout the US and elsewhere. Although $\mathrm{O}_{3}$ generally peaks in the summer throughout the US, for example, particulate matter peaks in the winter in California and in the summer on the East Coast. Thus, there would more likely be an impact of PM on elevated ambient temperature and health outcomes on the East Coast. Acclimatization may also play a critical role in the temperature-mortality association. People who live in areas where high ambient temperatures or heat waves are typically experienced may be less affected than people who reside in areas where high ambient temperatures are less commonly observed. Thus, even if there is effect modification between ambient temperature and a pollutant, such as $\mathrm{O}_{3}$, the influence on mortality may be minimal, but synergistic in areas where heat waves are uncommon.

Several vulnerable subgroups have been identified in the past decade of epidemiologic research, and were often dependent on study location and study population. Thus, region-specific policies, especially in urban areas, are vital to the mitigation of heat-related deaths. Specifically, those dying from cardiovascular, respiratory, and some specific cardiovascular diseases, such as ischemic heart disease, congestive heart failure, and myocardial infarction were at greater risk for heat-related mortality. Other vulnerable subgroups included: Black racial/ethnic group, women, those with lower socioeconomic status, and all age groups, particularly the elderly over 65 years of age as well as infants and young children.

Infants, young children, and the elderly should be specifically targeted in future studies to prevent heat-related mortality. With the elderly increasing in urban environments, an important research goal is the identification of clinical patterns of chronic diseases that increase the susceptibility to heat. Furthermore, vulnerable subgroups need to be further identified by cause-specific outcomes or demographics, such as racial/ethnic group. Furthermore, adverse birth outcomes have been found to be associated with air pollutants in previous studies, but have not been investigated, specifically for ambient temperature. Although previous studies of air pollution and birth outcomes have not accounted for temperature, some investigators have suggested seasonal associations, implying that temperature could also play a role with adverse birth outcomes and warrants further investigation.

Several biological mechanisms have been postulated for susceptible populations to heat-related mortality, particularly the elderly [42]. When body temperatures rise, blood flow generally shifts from the vital organs to underneath the skin's surface in an effort to cool down. The body's ability to regulate its temperature (also known as thermoregulation) may be impeded when too much blood is diverted, putting increased stress on the heart and lungs. Increased blood viscosity, elevated cholesterol levels associated with higher temperatures, and higher sweating threshold may also trigger heat-related mortality [43]. The body's ability to adapt to high ambient temperature can be influenced by acclimatization. People who live in areas where high ambient temperatures are not generally experienced are more likely to be affected by a heat wave. The synergistic impact of high ambient temperature along with high levels of air pollutants, such as $\mathrm{O}_{3}$ and $\mathrm{PM}$, may also play a role in increasing the mortality effect. Furthermore, heat waves occurring earlier in the year may have a greater impact on mortality since the population has not had the chance to adapt to hotter temperatures.

This review is timely as climate change receives more global attention, and more epidemiologic studies have been recently conducted. It, however, has several limitations. While it includes the most recent epidemiologic studies using time-series and case-crossover methods, it does not include studies of heat waves or studies using other approaches in an effort to focus on general ambient temperature over longer time periods. Both methods rely on ecologic exposure variables for temperature, and the timeseries analysis also uses aggregated counts of mortality. Thus, an advantage of the case-crossover study is that differences by individual-level characteristics such as age, race/ethnic group, gender can be analyzed. Although the methods used across studies were similar, it was still often difficult to compare estimates between studies because of the analysis type (e.g., different threshold values). There were also not a sufficient number of studies to conduct a meta-analysis of the results, or other more substantial quantification. Finally, there may be some publication bias in the studies that were chosen, but by using PubMed, the bias may be limited, as it includes most scientific journals.

Further studies need to be conducted in more urban locations so that policies can be implemented for specific 
areas rather than for an entire geographic area. These studies would be helpful to the National Weather Service, health care institutions, and governmental agencies to implement policies to prevent heat-related mortality and also create a better heat warning system based on current studies. They will also be helpful to establish policy guidelines for the U.S. Environmental Protection Agency (personal communication), and could be used for economic analyses. Although no formal evaluation of heat-health watch warning systems has been performed to date, some city-based heat-health watch warning systems have already been implemented appear to be successful in greatly reducing mortality following heat waves [44]. For example, the 2003 heat wave in Western Europe resulted in 35,000 deaths, but the World Health Organization's project, EuroHEAT, collected information about existing warning systems and defined guidelines for prevention so that subsequent heat waves do not produce such devastating results http://www.euro.who.int/document/ e91347.pdf.

\section{Conclusion}

In the past few years, several epidemiologic studies have been conducted in various locations to characterize temperature and mortality. These studies have consisted primarily of time-series and case-crossover studies, and were summarized this review. The recent epidemiologic evidence suggests that $\mathrm{PM}$ and $\mathrm{O}_{3}$ may be confounders, and some studies also found $\mathrm{O}_{3}$ to be an effect modifier in the warmer months. However, this confounding effect is relatively small, and there is clearly an independent effect of both temperature and air pollution on mortality. Several vulnerable subgroups have been identified, including those dying from cardiovascular, respiratory, and some specific cardiovascular diseases, such as ischemic heart disease, congestive heart failure, and myocardial infarction. Other vulnerable subgroups included: Black racial/ ethnic group, women, those with lower socioeconomic status, and all age groups, particularly the elderly over 65 years of age as well as infants and young children.

Thus, infants, young children, and the elderly should be specifically targeted in future studies to prevent heatrelated mortality. Many of these outcomes and vulnerable subgroups have only been identified in recent epidemiologic studies of ambient temperature and were dependent on the location and study population. Thus, region-specific policies, especially in urban areas, are vital to the mitigation of heat-related deaths.

\section{Abbreviations}

CO: (carbon monoxide); $\mathrm{NO}_{2}$ : (nitrogen dioxide); $\mathrm{O}_{3}$ : (ozone); PM: (particulate matter); $\mathrm{PM}_{2.5}$ : (particulate matter with less than $2.5 \mu \mathrm{g} / \mathrm{m}^{3}$ in aerodynamic diameter); $\mathrm{PM}_{10}$ : (particulate matter with less than $10 \mu \mathrm{g} / \mathrm{m}^{3}$ in aerodynamic diameter); $\mathrm{SO}_{2}:$ (sulfur dioxide); ${ }^{\circ} \mathrm{C}$ : (degree Celsius); ${ }^{\circ} \mathrm{F}$ : (degree Fahrenheit); CI: (confidence interval).

\section{Competing interests}

The author declares that they have no competing interests.

\section{Authors' contributions}

$\mathrm{RB}$ conducted the literature search for this review, specified the inclusion and exclusion criteria, constructed the tables, and drafted and revised the manuscript for consideration for publication.

\section{Acknowledgements}

The opinions expressed in this article are solely those of the author and do not represent the policy or position of the State of California or the California Environmental Protection Agency.

\section{References}

I. Basu R, Samet JM: Relation between elevated ambient temperature and mortality: a review of the epidemiologic evidence. Epidemiol Rev 2002, 24:190-202.

2. Kinney P, O'Neill MS, Bell ML, Schwartz J: Approaches for estimating effects of climate change on heat-related deaths: challenges and opportunities. Environmental Science \& Policy 2008, I I:87-96.

3. Gosling SN, McGregor GR, Paldy A: Climate change and heatrelated mortality in six cities part $I$ : model construction and validation. Int J Biometeorol 2007, 5 I:525-540.

4. Basu R, Samet JM: An exposure assessment study of ambient heat exposure in an elderly population in Baltimore, Maryland. Environ Health Perspect 2002, I I 0:1219-1224.

5. Basu R, Dominici F, Samet JM: Temperature and mortality among the elderly in the United States: a comparison of epidemiologic methods. Epidemiology 2005, 1 6:58-66.

6. Basu R, Feng WY, Ostro BD: Characterizing Temperature and Mortality in Nine California Counties. Epidemiology 2008, 19:138-|45.

7. Zanobetti A, Schwartz ]: Temperature and mortality in nine US cities. Epidemiology 2008, 19:563-570.

8. Braga AL, Zanobetti A, Schwartz J: The effect of weather on respiratory and cardiovascular deaths in 12 U.S. cities. Environ Health Perspect 2002, I I 0:859-863.

9. Zeger SL, Dominici F, Samet J: Harvesting-resistant estimates of air pollution effects on mortality. Epidemiology 1999, 10:171-175

10. Schwartz J: Harvesting and long term exposure effects in the relation between air pollution and mortality. $A m \mathrm{~J}$ Epidemiol 2000, I 5 I:440-448.

II. Braga AL, Zanobetti A, Schwartz J: The time course of weatherrelated deaths. Epidemiology 200I, I 2:662-667.

12. Baccini M, Biggeri A, Accetta G, Kosatsky T, Katsouyanni K, Analitis A, Anderson HR, Bisanti L, D'Ippoliti D, Danova J, et al.: Heat effects on mortality in I5 European cities. Epidemiology 2008, 19:711-719.

13. Hajat S, Kovats RS, Atkinson RW, Haines A: Impact of hot temperatures on death in London: a time series approach. J Epidemiol Community Health 2002, 56:367-372.

14. Basu R, Ostro BD: A multicounty analysis identifying the populations vulnerable to mortality associated with high ambient temperature in California. $A m J$ Epidemiol 2008, I 68:632-637.

I5. Bell ML, O'Neill MS, Ranjit N, Borja-Aburto VH, Cifuentes LA, Gouveia NC: Vulnerability to heat-related mortality in Latin America: a case-crossover study in Sao Paulo, Brazil, Santiago, Chile and Mexico City, Mexico. Int J Epidemiol 2008, 37:796-804

16. Stafoggia M, Forastiere F, Agostini D, Biggeri A, Bisanti L, Cadum E, Caranci N, de' Donato F, De Lisio S, De Maria M, et al.: Vulnerability 
to heat-related mortality: a multicity, population-based, case-crossover analysis. Epidemiology 2006, 17:3|5-323.

17. Rainham DG, Smoyer-Tomic KE: The role of air pollution in the relationship between a heat stress index and human mortality in Toronto. Environ Res 2003, 93:9-19.

18. O'Neill MS, Hajat S, Zanobetti A, Ramirez-Aguilar M, Schwartz J: Impact of control for air pollution and respiratory epidemics on the estimated associations of temperature and daily mortality. Int J Biometeorol 2005, 50:12I-129.

19. Vaneckova P, Beggs PJ, de Dear RJ, McCracken KW: Effect of temperature on mortality during the six warmer months in Sydney, Australia, between 1993 and 2004. Environ Res 2008 108:361-369.

20. Ren C, Tong S: Temperature modifies the health effects of particulate matter in Brisbane, Australia. Int J Biometeorol 2006, $51: 87-96$

21. Medina-Ramon M, Schwartz J: Temperature, Temperature Extremes, and Mortality: A Study of Acclimatization and Effect Modification in $\mathbf{5 0}$ United States Cities. Occup Environ Med 2007.

22. Ren C, Williams GM, Morawska L, Mengersen K, Tong S: Ozone modifies associations between temperature and cardiovascular mortality: analysis of the NMMAPS data. Occup Environ Med 2008, 65:255-260.

23. Filleul L, Cassadou S, Medina S, Fabres P, Lefranc A, Eilstein D, Le Tertre A, Pascal L, Chardon B, Blanchard $M$, et al:: The relation between temperature, ozone, and mortality in nine French cities during the heat wave of 2003. Environ Health Perspect 2006, I 1 4: 1344-1347.

24. Ishigami A, Hajat S, Kovats RS, Bisanti L, Rognoni M, Russo A, Paldy A: An ecological time-series study of heat-related mortality in three European cities. Environ Health 2008, 7:5.

25. Hajat S, Kovats RS, Lachowycz K: Heat-related and cold-related deaths in England and Wales: who is at risk? Occup Environ Med 2007, 64:93-100

26. Goodman PG, Dockery DW, Clancy L: Cause-specific mortality and the extended effects of particulate pollution and temperature exposure. Environ Health Perspect 2004, I I 2: 179-185

27. Medina-Ramón M, Zanobetti A, Cavanagh DP, Schwartz J: Extreme Temperatures and Mortality: Assessing Effect Modification by Personal Characteristics and Specific Cause of Death in a Multi-City Case-Only Analysis. Environ Health Perspect 2006, II 4: I33I.

28. Schwartz $\mathrm{j}$ : Who is sensitive to extremes of temperature?: $\mathbf{A}$ case-only analysis. Epidemiology 2005, 16:67-72.

29. Stafoggia M, Schwartz J, Forastiere F, Perucci CA: Does Temperature Modify the Association between Air Pollution and Mortality? A Multicity Case-Crossover Analysis in Italy. Am Epidemiol 2008, 167( ( 2): 1476-85.

30. Stafoggia $M$, Forastiere $F$, Agostini $D$, Caranci $N$, de'Donato $F$ Demaria M, Michelozzi P, Miglio R, Rognoni M, Russo A, Perucci CA Factors affecting in-hospital heat-related mortality: a multicity case-crossover analysis. J Epidemiol Community Health 2008, 62:209-215.

31. Dilaveris P, Synetos A, Giannopoulos G, Gialafos E, Pantazis A, Stefanadis C: CLimate Impacts on Myocardial infarction deaths in the Athens TErritory: the CLIMATE study. Heart 2006, 92: $|747-| 75 \mid$

32. Kolb S, Radon K, Valois MF, Heguy L, Goldberg MS: The short-term influence of weather on daily mortality in congestive heart failure. Arch Environ Occup Health 2007, 62:169-176.

33. Gouveia N, Hajat S, Armstrong B: Socioeconomic differentials in the temperature-mortality relationship in Sao Paulo, Brazil. Int J Epidemiol 2003, 32:390-397.

34. El-Zein A, Tewtel-Salem M, Nehme G: A time-series analysis of mortality and air temperature in Greater Beirut. Sci Total Environ 2004, 330:7I-80

35. Diaz J, Linares C, Tobias A: Impact of extreme temperatures on daily mortality in Madrid (Spain) among the 45-64 agegroup. Int J Biometeorol 2006, 50:342-348.

36. Hajat S, Armstrong B, Baccini M, Biggeri A, Bisanti L, Russo A, Paldy A, Menne B, Kosatsky T: Impact of High Temperatures on Mortality: Is There an Added Heat Wave Effect? Epidemiology 2006, 17:632-638.
37. O'Neill MS, Zanobetti A, Schwartz J: Disparities by race in heatrelated mortality in four US cities: the role of air conditioning prevalence. J Urban Health 2005, 82: 191-197.

38. O'Neill MS, Zanobetti A, Schwartz J: Modifiers of the temperature and mortality association in seven US cities. Am J Epidemiol 2003, I57:1074-1082.

39. Curriero FC, Heiner KS, Samet JM, Zeger SL, Strug L, Patz JA: Temperature and mortality in II cities of the eastern United States. Am J Epidemiol 2002, 155:80-87.

40. Kim H, Ha JS, Park J: High temperature, heat index, and mortality in 6 major cities in South Korea. Arch Environ Occup Health 2006, 61:265-270

4I. Blanchard C: Spatial and Temporal Characterization of Particulate Matter. In Particulate Matter Science for Policy Makers: A NARSTO Assessment Volume Chapter 6. Edited by: McMurry PH, Shepherd MF, Vickery JS. Cambridge: Cambridge University Press; 2003.

42. Bouchama A, Knochel JP: Heat stroke. N Engl J Med 2002 346: $1978-1988$

43. Astrand P-O, Rodahl K, Dahl HA, Stromme SB: Textbook of Work Physiology: Physiological Bases of Exercise 4th edition. Canada: McGraw-Hill; 2003

44. Smoyer KE, Rainham DG, Hewko JN: Heat-stress-related mortality in five cities in Southern Ontario: 1980-1996. Int J Biometeorol 2000, 44: 190-197.

45. McMichael AJ, Wilkinson P, Kovats RS, Pattenden S, Hajat S, Armstrong B, Vajanapoom N, Niciu EM, Mahomed H, Kingkeow C, et al:: International study of temperature, heat and urban mortality: the 'ISOTHURM' project. Int J Epidemiol 2008, 37: | | 2 | - I | 3 |.

46. Vaneckova P, Hart MA, Beggs PJ, de Dear RJ: Synoptic analysis of heat-related mortality in Sydney, Australia, 1993-200 I. Int J Biometeorol 2008, 52:439-45I.

47. Barnett AG: Temperature and cardiovascular deaths in the US elderly: changes over time. Epidemiology 2007, 18:369-372.

48. Hajat SA, Ben G, Nelson Gouveia, Paul Wilkinson: Mortality Displacement of Heat-Related Deaths: A Comparison of Delhi, Sao Paulo, and London. Epidemiology 2005, 16:613.

49. Michelozzi P, De Sario M, Accetta G, de'Donato F, Kirchmayer U, D'Ovidio M, Perucci CA: Temperature and summer mortality: geographical and temporal variations in four Italian cities. J Epidemiol Community Health 2006, 60:417-423.

50. Pattenden S: Mortality and temperature in Sofia and London. 2003.

51. Ren C, Williams GM, Tong S: Does particulate matter modify the association between temperature and cardiorespiratory diseases? Environ Health Perspect 2006, II 4:1690-1696.

52. Yip FY, Flanders WD, Wolkin A, Engelthaler D, Humble W, Neri A, Lewis $L$, Backer $L$, Rubin C: The impact of excess heat events in Maricopa County, Arizona: 2000-2005. Int / Biometeorol 2008, 52:765-772.

Publish with Biomed Central and every scientist can read your work free of charge

"BioMed Central will be the most significant development for disseminating the results of biomedical research in our lifetime. "

Sir Paul Nurse, Cancer Research UK

Your research papers will be:

- available free of charge to the entire biomedical community

- peer reviewed and published immediately upon acceptance

- cited in PubMed and archived on PubMed Central

- yours - you keep the copyright 